\title{
Shell-Filling Effects in Circular Quantum Dots
}

\author{
M. MACUCCI ${ }^{\mathrm{a}, *}$ and KARL HESS ${ }^{\mathrm{b}}$ \\ ${ }^{a}$ Dipartimento di Ingegneria dell' Informazione, Via Diotisalvi, 2 I-56126 Pisa, Italy; \\ ${ }^{\mathrm{b}}$ Beckman Institute, $405 \mathrm{~N}$ Mathews, Urbana, IL 61801
}

\begin{abstract}
We investigate the dependence of the capacitive energy associated with a circular quantum dot on the number of electrons in the dot and on the parameters defining the potential confinement. Our results reproduce the shell-filling behavior that has recently been experimentally observed and allow us to estimate the shape of the confinement potential and the dot size of the experiments.
\end{abstract}

Keywords: Quantum dot, shell-filling, charging energy, density functional

\section{INTRODUCTION}

Recent advances in nanofabrication techniques have allowed a more detailed investigation of the electronic structure of quantum dots. In particular, it has become possible to measure, with greater precision and reliability, the values for the charging energies in a quantum dot. Tarucha et al. [1] have fabricated a circular quantum dot in which vertical confinement has been achieved by means of $\mathrm{AlGaAs}$ barriers $12 \mathrm{~nm}$ apart, while lateral confinement has been obtained by etching and by means of the depletion due to a lateral Schottky contact. Tarucha et al. have evaluated the charging energies, which they improperly name "addition energies", by measuring the $I-V$ characteristic for several different values of the gate voltage: in this way it is possible to construct the "diamond diagram", typical of Coulomb blockade studies, which yields directly the values for the charging energies.

For the first time, the results in Ref. [1] show atomic-like shell-filling effects in a quantum dot, as predicted in Refs. [2], [4]. In order to understand the meaning of these results, we must first define rigorously what the charging energy is. We can start from the classical definition of the capacitive energy, i.e., the variation in the potential energy of a capacitor as a consequence of the addition of charge. For a quantum dot the charging energy can be defined as the variation of the chemical potential as a consequence of the addition of one electron: $E_{C}=\mu(N+1)-\mu(N)$. If the dot were a classical capacitor, this would be a constant

\footnotetext{
* Corresponding author.
} 
quantity; in the quantum case we can define a quantum capacitance [2], depending on the number $N$ of electrons contained in the dot, as $C(N)=e^{2} / E_{C}$, where $e$ is the charge of the electron.

As long as we add electrons to the same orbital, the behavior of the quantum dot is quite similar to that of a classical capacitor: the charging energy is substantially constant and depends mostly on the geometrical dimensions. However, when we start populating a new orbital, the charging energy exhibits a peak, due to the variation in the quantum confinement energy. Thus, the charging energy is about constant, with a peak every time that a new shell opens. In a rectangular dot, for example, each orbital accomodates only two electrons, so that the charging energy has an oscillating behavior, as a function of the electron number. A circular dot, instead, has several degeneracies, which lead to a much smaller number of peaks, as will be discussed in the following.

\section{MODEL}

We have considered a 2D model of a quantum dot characterized by parabolic confinement and hard walls located at a distance from the center corresponding to the geometrical radius. For purposes of easy comparison with the experimental results, we assume the parabolic potential given by $V=1 / 2 m^{*} \omega^{2} r^{2}$, where $r$ is the radial coordinate and $m^{*}$ is the electron effective mass, and express the slope of such potential by means of the product $\hbar \omega, \hbar$ being the reduced Planck constant. The 2D approximation is allowed by the fact that the vertical dimension of the $\operatorname{dot}(12 \mathrm{~nm})$ is much smaller than the lateral radius, therefore only the lowest state along the vertical direction is occupied, and this represents just a constant shift in the energy, which does not affect the results for the charging energy. The 2D Schrödinger equation can be separated [3] into a straightforward angular equation and into a radial equation, which is solved by means of a standard numerical finite- difference procedure. The two equations read:

$$
\begin{gathered}
\frac{d^{2} \Phi(\phi)}{d \phi^{2}}+\nu^{2} \Phi(\phi)=0 \\
\frac{d^{2} P(\rho)}{d \rho^{2}}+\frac{1}{\rho} \frac{d P(\rho)}{d \rho^{2}}-\frac{2 m^{*}}{\hbar^{2}} V(\rho) P(\rho) \\
+\left(k^{2}-\frac{\nu^{2}}{\rho^{2}}\right) P(\rho)=0
\end{gathered}
$$

where $\nu^{2}$ is the constant for the separation of variables and $k^{2}=2 m^{*} E / \hbar^{2}, E$ being the energy eigenvalue. If we enforce periodic boundary conditions, the angular equation has the simple solution $\Phi=c \exp ( \pm i \phi)$. When $\nu^{2}=0$, there is just one solution, while for $\nu^{2} \neq 0$ two different degenerate solutions exist, corresponding to the up and down orientations of the angular momentum.

The potential $V(\rho)$ is computed self-consistently at each iteration from the electron density obtained at the previous iteration. It includes also a term accounting for the exchange and correlation interactions, in the framework of a local density functional approach [3]. The iterative procedure is stopped when the electron density differs from that of the preceding iteration less than a given amount. Obtaining convergence is simple as long as the Coulomb interaction among the electrons represents just a perturbation of the quantum confinement energy. This condition is satisfied for very small dots, since the electrostatic interaction energy scales with the inverse of the distance, while the quantum confinement energy scales with the inverse of the square of the dot size. Thus, for dots larger than about $100 \mathrm{~nm}$, particular care has to be taken to ensure convergence, with the usage of underrelaxation techniques, which help controlling the instabilities typical of fixedpoint iteration schemes.

The chemical potential is obtained applying Slater's transition rule [3], which allows better numerical precision than that obtainable from a differentiation of the total energy. 


\section{NUMERICAL RESULTS AND DISCUSSION}

The reason why we have chosen the particular potential landscape described in the previous section is that it qualitatively explains a feature observed in the experimental results: the charging energy tends to saturate to a limiting value as if the size of the dot were growing while the number of electrons is increased, until any further growth is limited by the presence of hard walls.

We have first considered a quantum dot with a geometrical radius of $90 \mathrm{~nm}$ and we have computed the capacitive energy for three different slopes of the parabolic confinement potential. The results are reported in Figure 1, where the charging energy is plotted versus the number of electrons in the dot for $\hbar \omega=4 \mathrm{meV}$ (thick line), $3 \mathrm{meV}$ (thin line) and 2.5 $\mathrm{meV}$ (dashed line). In all three cases the capacitive energy tends to decrease and to saturate down to a limiting value. This is the consequence of the variation of the effective dot size (defined as the area over which the electron density is nonnegligible) as a function of the number of electrons:.when there are just a few electrons, they tend to concentrate in the deepest region of the potential landscape, near the dot center, while for larger numbers of electrons such minimum is effectively screened and the charge is distributed over a larger area, up to the maximum size allowed by the hard walls. The classical charging energy $E_{c c}$ for a

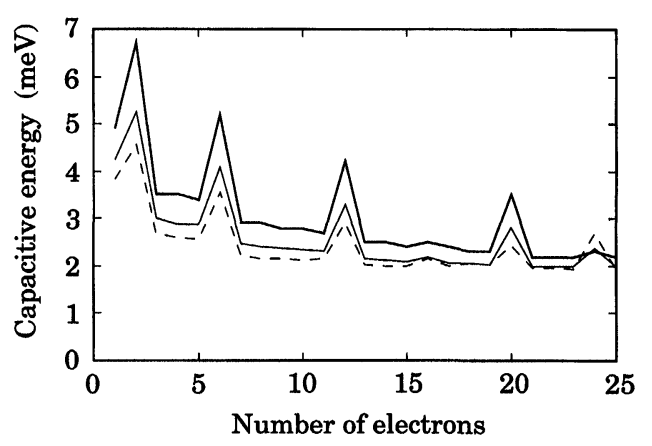

FIGURE 1 Charging energy for a quantum dot with a radius of $90 \mathrm{~nm}$ versus the number of electrons. The bare confinement potential is parabolic with $\hbar \omega=4 \mathrm{meV}$ (thick line), $3 \mathrm{meV}$ (thin line), $2.5 \mathrm{meV}$ (dashed line). conducting disk with a radius of $90 \mathrm{~nm}$ is given [5] by $E_{c c}=e^{2} /\left(8 R \varepsilon_{0} \varepsilon_{r}\right)=1.94 \mathrm{meV}$, where $R$ is the disk radius, $\varepsilon_{0}$ is the vacuum permittivity and $\varepsilon_{r}$ is the relative permittivity of the medium. This is in good agreement with the limiting value of approximately $2 \mathrm{meV}$ obtained from the simulation. Another major feature of the computed charging energy consists in the presence of peaks for $N=2,6,12,16$, 20,24 , corresponding to those found in the experimental results. Each of them is associated with the filling of a shell (orbital) and its height depends on the difference between the energy eigenvalue of the new shell and that of the previous one. If the confinement potential were perfectly parabolic, without any contribution from electronelectron interaction, there would be peaks only for $N=2,6,12,20,30$, because more degeneracies are present with a parabolic potential than with a generic potential characterized by circular symmetry. Circular symmetry only implies that when $\nu=0$ there is a double degeneracy (the spin degeneracy) and when $\nu \neq 0$ the degeneracy is fourfold (spin degeneracy plus angular momentum degeneracy). We do not observe any peak for $N=10$, because the energy splitting between the two related orbitals is extremely small (see Ref. [6] for more details).

The saturation of the charging energy towards a limiting value determined by the maximum dot size is apparent in Figure 2, where we report the capacitive energies for $\hbar \omega=3 \mathrm{meV}$ and a dot radius of $75 \mathrm{~nm}$ (thick line), $90 \mathrm{~nm}$ (thin line) and $120 \mathrm{~nm}$ (dashed line). When the number of electrons in the dot is small, the effective size is independent of the geometrical radius and is determined only by the shape of the parabolic potential, therefore for small $N$ the three curves overlap. For large numbers of electrons, instead, the three curves tend to saturate to the corresponding classical capacitive energies (the curve for $R=120 \mathrm{~nm}$ does not reach the limiting value for the maximum number of electrons included in our plot).

The experimental results by Tarucha et al. show a saturation value of approximately $1.25 \mathrm{meV}$, which would correspond to a classical radius of 


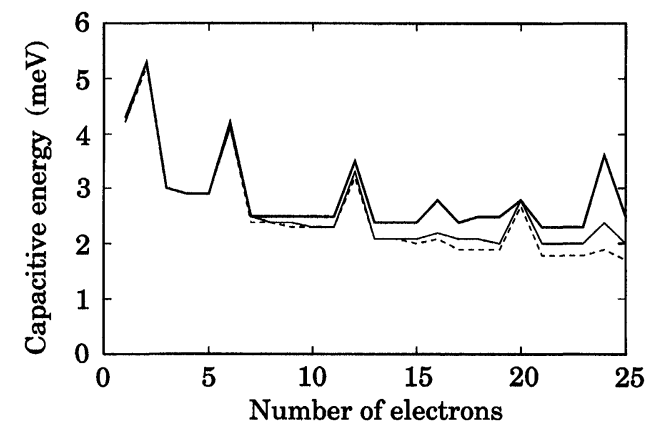

FIGURE 2 Charging energy for a quantum dot with a parabolic confinement potential defined by $\hbar \omega=3 \mathrm{meV}$ and with a geometrical radius of $75 \mathrm{~nm}$ (thick line), $90 \mathrm{~nm}$ (thin line) and $120 \mathrm{~nm}$ (dashed line).

$140 \mathrm{~nm}$. We have therefore performed a calculation for a dot with a geometrical radius of $140 \mathrm{~nm}$, choosing a confinement potential with $\hbar \omega=2.48$ $\mathrm{meV}$, on the basis of the best possible agreement between the results of the simulation and the experimental data. The computed capacitive energy as a function of the electron number is reported in Figure 3: the only significant discrepancy with the experimental results is represented by the absence of the peak for $N=4$, which cannot be explained if we assume rigorous circular symmetry. It is however possible that some irregularity exists in the confinement potential of the experimental dot and that it produces measurable effects only for small numbers of electrons, while it is screened out for larger values of $N$.

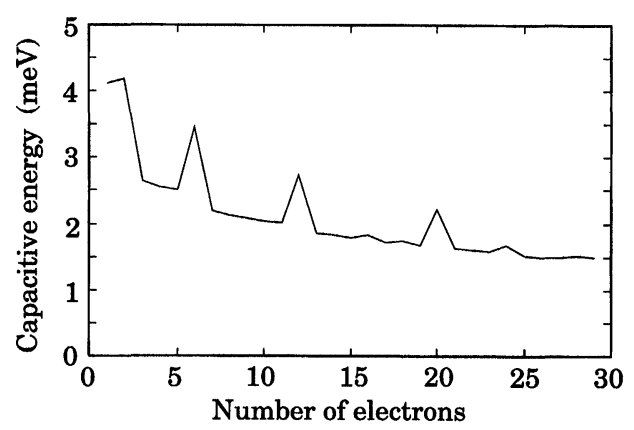

FIGURE 3 Charging energy for a quantum dot with a parabolic confinement potential defined by $\hbar \omega=2.48 \mathrm{meV}$ and with a geometrical radius of $140 \mathrm{~nm}$.

\section{CONCLUSIONS}

The charging energy for a circular model quantum dot has been evaluated with the inclusion of exchange and correlation effects. The numerical results are in quantitative agreement with experimental data that have recently been obtained and reproduce the position of the observed peaks, corresponding to shell-filling events.

\section{Acknowledgements}

This work has received partial support from the NATO Collaborative Research Grant n. 950753. One of the authors (M. M.) acknowledges support from the Italian Ministry of the University and Scientific Research. K. H. acknowledges the support of the US Army Research Office.

\section{References}

[1] Tarucha, S., Austing, D. G., Honda, T., van der Hage, R. J. and Kouwenhoven, L. P. (1996). "Shell Filling and Spin Effects in a Few Electron Quantum Dot", Phys. Rev. Lett., 77, 3613.

[2] Macucci, M., Hess, K. and Iafrate, J. G. (1993). "Electronic Energy Spectrum and the Concept of Capacitance in Quantum Dots", Phys. Rev. B, 48, 17354.

[3] Macucci, M., Hess, K. and Iafrate, G. J. (1995). "Simulation of electronic properties and capacitance of quantum dots", J. Appl. Phys., 77, 3267.

[4] Iafrate, G. J., Hess, K., Krieger, J. B. and Macucci, M. (1995). "Capacitive nature of atomic-sized structure", Phys. Rev. B, 52, 10737.

[5] van Houten, H., Beenakker, C. W. J. and Staring, A. A. M. (1992). In "Single Charge Tunneling", edited by Grabert, H. and Devoret, M. H. (Plenum Press, New York).

[6] Macucci, M., Hess, K. and Iafrate, G. J. (1997). "Numerical simulation of shell-filling effects in circular quantum dots", Phys. Rev. B, 55, R4879.

\section{Authors' Biographies}

Massimo Macucci is serving on the faculty of the Electrical Engineering Department at the University of Pisa, Italy. His research interests include quantum-interference and single-electron devices, Coulomb Blockade phenomena, modeling and measurements of noise in electron devices. 
Karl Hess holds the Swanlund Chair in Electrical and Computer Engineering at the University of Illinois in Urbana-Champaign. His main inter- ests are in computational electronics, optoelectronics and most recently in the physics of semiconductor nanostructures. 

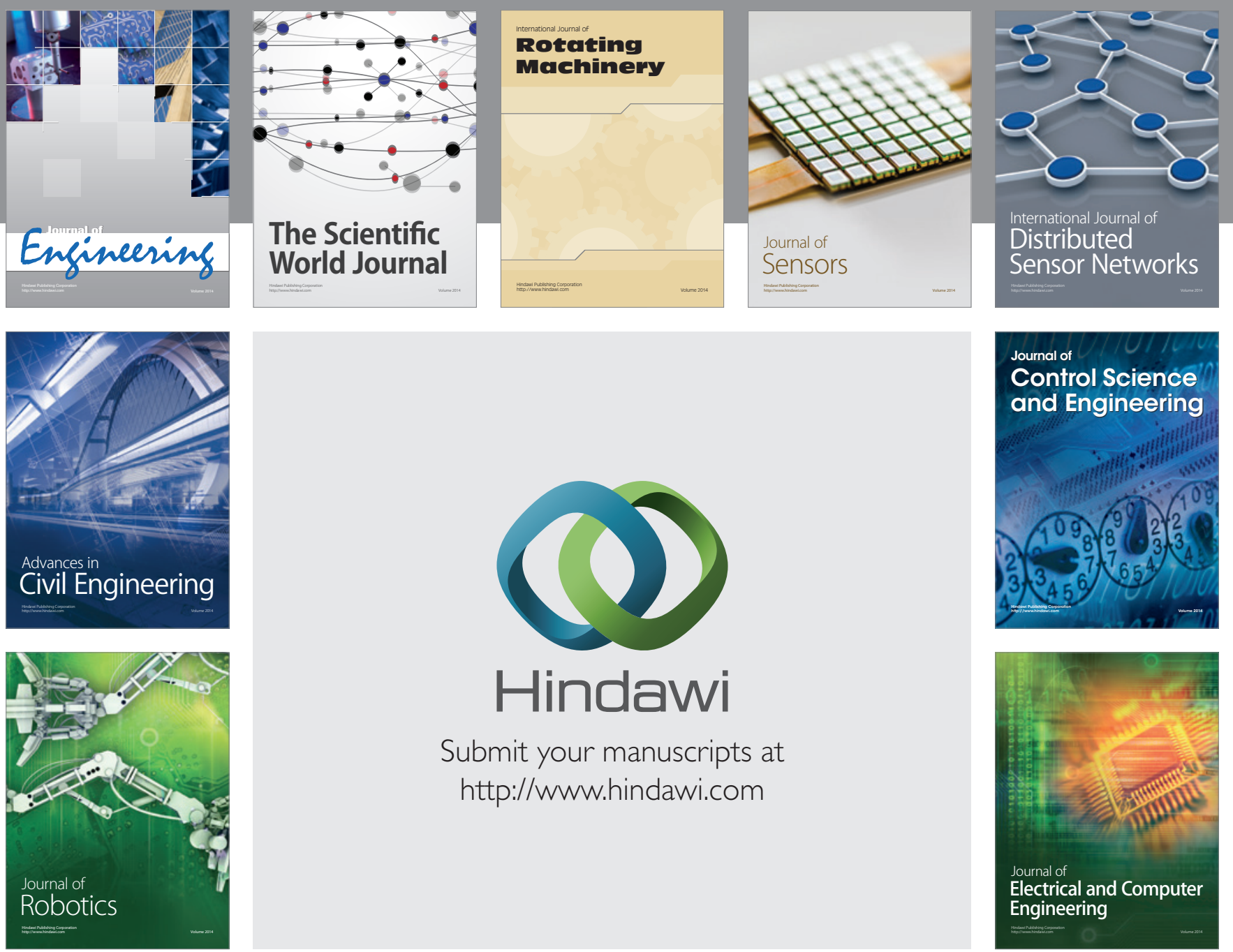

Submit your manuscripts at

http://www.hindawi.com
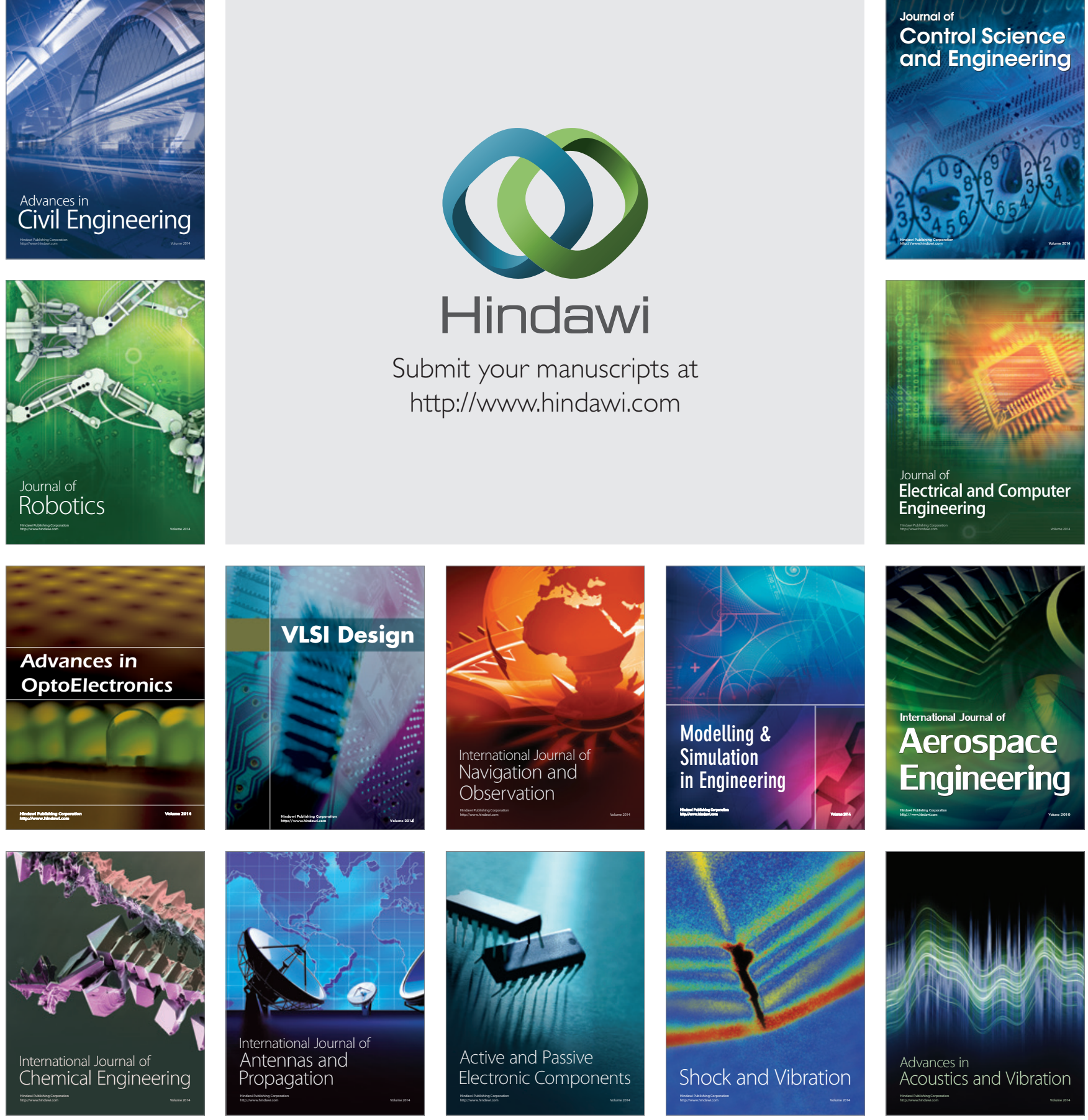